University of Wollongong

Research Online

Faculty of Engineering and Information

Faculty of Engineering and Information

Sciences - Papers: Part A

Sciences

$1-1-2016$

Cubic autocatalysis in a reaction-diffusion annulus: semi-analytical solutions

Muteb Alharthi

University of Taif, mraah761@uowmail.edu.au

Timothy R. Marchant

University of Wollongong, tim@uow.edu.au

Mark Nelson

University of Wollongong, mnelson@uow.edu.au

Follow this and additional works at: https://ro.uow.edu.au/eispapers

Part of the Engineering Commons, and the Science and Technology Studies Commons

Research Online is the open access institutional repository for the University of Wollongong. For further information contact the UOW Library: research-pubs@uow.edu.au 


\title{
Cubic autocatalysis in a reaction-diffusion annulus: semi-analytical solutions
}

\author{
Abstract \\ Semi-analytical solutions for cubic autocatalytic reactions are considered in a circularly symmetric \\ reaction-diffusion annulus. The Galerkin method is used to approximate the spatial structure of the \\ reactant and autocatalyst concentrations. Ordinary differential equations are then obtained as an \\ approximation to the governing partial differential equations and analyzed to obtain semi-analytical \\ results for this novel geometry. Singularity theory is used to determine the regions of parameter space in \\ which the different types of steady-state diagram occur. The region of parameter space, in which Hopf \\ bifurcations can occur, is found using a degenerate Hopf bifurcation analysis. A novel feature of this \\ geometry is the effect, of varying the width of the annulus, on the static and dynamic multiplicity. The \\ results show that for a thicker annulus, Hopf bifurcations and multiple steady-state solutions occur in a \\ larger portion of parameter space. The usefulness and accuracy of the semi-analytical results are \\ confirmed by comparison with numerical solutions of the governing partial differential equations.

\section{Disciplines} \\ Engineering | Science and Technology Studies

\section{Publication Details} \\ Alharthi, M. R., Marchant, T. R. \& Nelson, M. I. (2016). Cubic autocatalysis in a reaction-diffusion annulus: \\ semi-analytical solutions. Zeitschrift fur Angewandte Mathematik und Physik, 67 (3), 65-1 - 65-13.
}




\title{
Cubic-autocatalysis in a reaction-diffusion annulus: semi-analytical solutions
}

\author{
M. R. Alharthi ${ }^{1}$ and T. R. Marchant ${ }^{2}$ and M. I. Nelson ${ }^{2}$ \\ ${ }^{1}$ Mathematics and Statistics Department, \\ Faculty of Science, University of Taif, \\ Taif, Saudi Arabia \\ 2 School of Mathematics and Applied Statistics \\ The University of Wollongong, Wollongong, 2522, N.S.W., Australia. \\ email: tim_marchant@uow.edu.au
}

\begin{abstract}
Dedication: This paper is dedicated to Professor James Hill on the occasion of his 70th birthday. Prof. Hill has been a source of inspiration to the authors, over many years, due to his significant contributions to Applied Mathematics and leadership of the field within Australia. He has also played a key role in the support and mentoring of a new generation of Applied Mathematicians that will influence the field for many years to come.
\end{abstract}

\begin{abstract}
Semi-analytical solutions for cubic autocatalytic reactions are considered in a circularly symmetric reactiondiffusion annulus. The Galerkin method is used to approximate the spatial structure of the reactant and autocatalyst concentrations. Ordinary differential equations are then obtained as an approximation to the governing partial differential equations and analyzed to obtain semi-analytical results for this novel geometry. Singularity theory is used to determine the regions of parameter space in which the different types of steady-state diagram occur. The region of parameter space, in which Hopf bifurcations can occur, is found using a degenerate Hopf bifurcation analysis. A novel feature of this geometry is the effect, of varying the width of the annulus, on the static and dynamic multiplicity. The results show that for a thicker annulus, Hopf bifurcations and multiple steady-state solutions occur in a larger portion of parameter space. The usefulness and accuracy of the semi-analytical results are confirmed by comparison with numerical solutions of the governing partial differential equations.
\end{abstract}

Mathematics Subject classifications. 35A35, 37C75, 80A30.

Keywords. reaction-diffusion equations; Gray-Scott scheme; singularity theory; Hopf bifurcations; semi-analytical solutions.

\section{Introduction}

Many chemical systems display oscillatory solutions and multiple steady-state solutions; examples include the BelousovZhabotinskii (BZ), Bray-Liebhafsky and Briggs-Rausher systems. These systems undergo periodic concentration variations and have the added interest that these oscillations can be visualized via colour changes, see Corbel et al. [1]. A wide range of phenomena is possible including multi-stability, chaos, bursting, reaction-diffusion patterns and waves and feedback control, see Sagues and Epstein [2] for a comprehensive review of these phenomena for chemical systems.

Gray-Scott is a classical theoretical scheme, which represents cubic autocatalysis with linear catalyst decay. It has been widely considered over many years, because of its variety of steady-state responses and oscillatory solutions. The scheme is

$$
A+2 B \rightarrow 3 B, \quad \text { rate }=\beta \mathrm{ab}^{2}, \quad \mathrm{~B} \rightarrow \mathrm{C}, \quad \text { rate }=\beta \gamma \mathrm{b},
$$

where the concentrations of the reactant and autocatalyst are $a$ and $b$, respectively. The catalyst is not stable, but undergoes a simple linear decay. This generates a much wider variety of behavior than does the cubic reaction alone. Gray and Scott $[3,4]$ analyzed the cubic autocatalytic reaction (1) in a continuous stirred tank reactor (CSTR). They found that the model has three types of steady-state bifurcation diagrams (the unique, mushroom and isola patterns). They also mapped the parameter region where Hopf bifurcations occur. Numerical simulations of the governing ordinary differential equations (odes) showed the evolution of the system to stable or unstable limit-cycles and oscillatory decay to a stable steady state. Kay et al. [5] showed that, when an uncatalysed conversion step is added, the number of steady-state diagrams increases to five. However, the new breaking-wave and isola breaking-wave patterns only occurred in very small regions of parameter space.

In the non-stirred case chemical systems are governed by a system of partial differential equations (pdes). Scott [6] and Kay and Scott [7] obtained numerical results for the Gray-Scott reaction-diffusion cell and identified four steadystate patterns. Again a Hopf-bifurcation parameter map was drawn. Marchant [8] considered semi-analytical solutions for the Gray-Scott reaction-diffusion cell. The Galerkin method was used to obtain a lower-order ode model, as an approximation to the governing pde system. Semi-analytical bifurcation patterns and Hopf bifurcation parameter maps were found. A good comparison was found between the results of the semi-analytical method and the numerical solutions of the governing pdes. Extensive experimental work has also been performed using reaction-diffusion cells. An inert gel medium is used which prevents convective motion but allows diffusion of the chemical species. The gel medium is coupled to a CSTR hence the boundary concentrations can be controlled via flow of reactants in the CSTR. Tam et 
al. [9] considered an experimental reaction-diffusion cell to analyse the spatial patterns forming for a BZ reaction while Bagyan et al. [10] and Lavrova et al. [11] considered glycolytic reactions using a similar experimental set-up.

Cylindrical and spherical geometries are also used to model many processes governed by reaction-diffusion systems, such as tumor growth and tissue development via morphogenesis, see [12, 13, 14]. Protein trafficking in cells was modelled in a two-dimensional reaction-diffusion annulus, see Jiangguo and Simon [15]. Moreover, the reaction-diffusion annulus is a novel geometry used to consider rotating chemical waves. Farr and Golubitsky [16] considered the Gray-Scott model in a circular geometry, and the stability of rotating wave solutions that formed via Hopf bifurcations. It was found that stable rotating waves exist over broad ranges of parameter values and that the bifurcation behavior of this relatively simple model can be quite complex, with two- and three-frequency motions existing. Lubkin and Rand [17] studied the behavior of rotating spiral waves in a reaction-diffusion circular disk. They found a condition for the existence of spiral waves and also studied the effect of diffusivity and disk size on the properties of the spiral waves. Bar et al. [18] considered a simple activator-inhibitor model in a circular domain and considered the bifurcation and stability analysis of rotating spiral waves numerically. This study showed that the domain size affects the stability of this model, in small domains the waves are subject to a meandering instability while in large domains the meandering instability is suppressed and at a critical radius the spiral rotation became rigid. Tsai [19] investigate how the diffusivity, the domain size and the reaction kinetics affect the properties of spiral waves in a reaction-diffusion disk with a no-flux boundary condition. The study showed that the rotational frequency increases with increasing domain size.

In this paper, the Gray-Scott scheme (1) is examined in a circularly symmetric annulus. The Galerkin method is used to apply the semi-analytical method to the annulus geometry. This is one of the first studies to consider reactions in an annulus geometry and allows us to explore geometric effects on the static and dynamic stability. In $\S 2$ the governing equations are presented and the Galerkin method is used to obtain the odes which represent the semi-analytical model. In $\S 3$ both steady-state concentration profiles and bifurcation patterns are presented and described in detail. In $\S 4$ singularity theory is used to calculate the hysteresis and isola bifurcation points. The loci of these singularities are plotted and the regions of parameter space are identified in which the four generic steady-state diagrams occur. In $\S 5$ a local stability analysis of the semi-analytical model is performed. The double-zero eigenvalue and transversally degenerate Hopf points are found; hence the parameter region in which Hopf bifurcations occur is identified. A key focus is the effect of varying the width of the annulus, on the static and dynamic stability. Comparisons are made between the semi-analytical results and numerical solutions of the governing pdes. Appendix A details the ode model for a special case.

\section{The semi-analytical model}

\subsection{The governing equations}

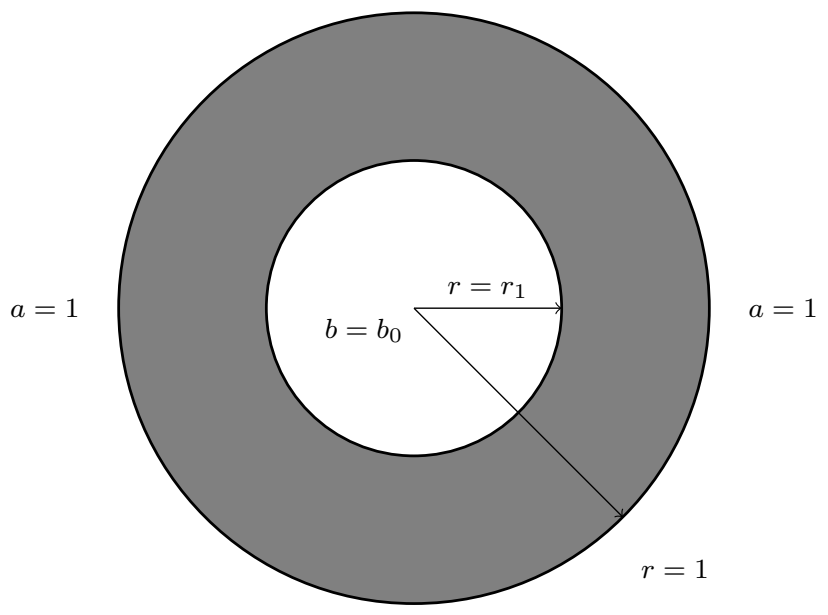

FiguRE 1. The geometry of the reaction-diffusion annulus, $a$ is the reactant and $b$ is the autocatalyst. 
The Gray-Scott scheme (1) is considered in a circularly symmetric annulus. The governing pdes are

$$
\begin{aligned}
a_{t} & =a_{r r}+\frac{1}{r} a_{r}-\beta a b^{2}, \quad b_{t}=b_{r r}+\frac{1}{r} b_{r}+\beta a b^{2}-\beta \gamma b, \\
a & =1, b=0 \text { at } \mathrm{r}=1 \text { and } \mathrm{a}=0, \mathrm{~b}=\mathrm{b}_{0} \text { at } \mathrm{r}=\mathrm{r}_{1}, \mathrm{r}_{1} \leqslant \mathrm{r} \leqslant 1 . \\
a & =1-\frac{\ln r}{\ln _{1}}, b=b_{0} \frac{\ln r}{\operatorname{lnr}_{1}} \text { at } \mathrm{t}=0 .
\end{aligned}
$$

The system (2) is in non-dimensional form with the concentrations of the reactant and autocatalyst given by a and b, respectively. It is an open system; the annulus has permeable boundaries at $r=1$ and $r=r_{1}$, joined to reservoirs which contains $a$ and $b$ at constant concentrations. This geometry allow the influx of the reactant at the outer boundary and the autocatalyst at the inner boundary. Experimentally this can be achieved using a diffusive gel coupled to CSTR's, which represents the inner and outer reservoirs, see [9, 10, 11]. By using high flow rates the concentrations in the CSTR's remain close to the input values as any reactions can be neglected.

A novel aspect of this geometry is the ability to vary the inner radius of the annulus. Note that $r_{1} \in(0,1)$, and as $r_{1} \rightarrow 0$, a disk is obtained. In this limit there is no inner reservoir and hence no supply of autocatalyst b. In the limit $r_{1} \rightarrow 1$, the annulus is vanishing thin. The initial condition satisfies the boundary conditions and is the steady-state solution of the system when there are no reaction terms (when $\beta=0$ ). The system is characterized by four non-dimensional parameters; $b_{0}$ is the autocatalyst concentration in the reservoir, $\beta$ is a measure of the importance of the reaction terms, $\gamma$ is a measure of the importance of autocatalyst decay, and $r_{1}$ is the radius of the inner edge of the annulus, as shown in figure 1 . Two ways to adjust the non-dimensional parameters experimentally would be to vary the reservoir concentration $b_{0}$ or alter the inner radius $r_{1}$.

\subsection{The Galerkin method}

The Galerkin method assumes the spatial structure of the concentration profiles, which then allows the governing pdes (2) to be approximated by odes. The method requires that the concentrations be approximated by a series of orthogonal basis functions. The expansion

$$
\begin{aligned}
a(r, t) & =1-\frac{\ln r}{\ln r_{1}}+a_{1}(t) \sin \theta_{1}+a_{2}(t) \sin 2 \theta_{1} \\
b(r, t) & =b_{0} \frac{\ln r}{\ln r_{1}}+b_{1}(t) \sin \theta_{1}+b_{2}(t) \sin 2 \theta_{1}, \theta_{1}=\frac{\pi\left(r-r_{1}\right)}{1-r_{1}}
\end{aligned}
$$

represents the two-term method used here. The trial functions include the steady-state solution of the governing equations without reaction terms, as these are needed to satisfy the boundary conditions. Expansion (3) satisfies the boundary conditions in (2), but not the governing pdes. The free parameters in (3) are found by evaluating averaged versions of the governing equations, weighted by the basis functions, which are $\sin \theta_{1}$ and $\sin 2 \theta_{1}$. This procedure gives a system of odes for any fixed value of $r_{1}$.

In the Appendix, equations (12)-(15) show the ode system when $r_{1}=0.25$. For a given value of inner radius $r_{1}$ the ode model is relatively straightforward to analyse. However, the reactor geometry is not fixed, so as $r_{1}$ varies, the range of integration changes and the coefficients must be numerically recalculated for each value of $r_{1}$. Hence many different ode models must be considered to examine the effect of varying the inner radius $r_{1}$.

\section{Steady-state solutions}

The steady-state versions of the odes are represented by a set of four transcendental equations, which are solved numerically using Maple. Figure 2 shows steady-state concentration profiles of the reactant and the autocatalyst, $a$ and $b$, versus $r$ for (a) $\beta=40$ and (b) $\beta=2000$. The parameters are $r_{1}=0.5, b_{0}=0.2$ and $\gamma=0.05$. The concentrations are shown at the centre of the annulus,

$$
a=1-\frac{\ln r_{c}}{\ln r_{1}}+a_{1}, \quad b=\frac{b_{0} \ln r_{c}}{\ln r_{1}}+b_{1}, \quad r_{c}=\frac{1+r_{1}}{2}
$$

The two-term semi-analytical and numerical solutions of (2) are shown. The concentration profiles are monotonic and qualitatively similar to the diffusion only (when $\beta=0$ ) $\ln r$ type profiles. It can be seen that the two-term expression gives a good approximation to the numerical solution of the governing pdes. At the centre of the annulus, $r=r_{c}$, the errors are only about $1 \%$. In figure $2(\mathrm{a})$, when $\beta$ is small, the steady-state concentration profiles are close to the diffusion only profile but, for larger $\beta$, as shown in figure $2(\mathrm{~b})$, the concentration profiles deviate from this $\ln r$ type profile. For example, in figure 2 the autocatalyst concentration at $r_{c}$ is $b=0.021$ compared to $b=0.082$ for a diffusion only system.

Figure 3 shows steady-state diagrams for the four generic patterns for $r_{1}=0.1$. They are the (a) unique $\left(b_{0}=0.3\right.$ and $\gamma=0.02)$, (b) breaking-wave $\left(b_{0}=0.1\right.$ and $\left.\gamma=0.01\right),(\mathrm{c})$ mushroom $\left(b_{0}=0.12\right.$ and $\left.\gamma=0.018\right)$ and (d) isola $\left(b_{0}=0.03\right.$ and $\left.\gamma=0.015\right)$ patterns. The steady-state diagrams show the autocatalyst concentration $b$ at the centre of the annulus, $r=r_{c}$, versus the bifurcation parameter $\beta$. The two-term semi-analytical solutions plus the numerical solution of (2) are shown. There are Hopf points when $\beta=980$ and 524 in figure 3(c) and 3(d) respectively, so no 


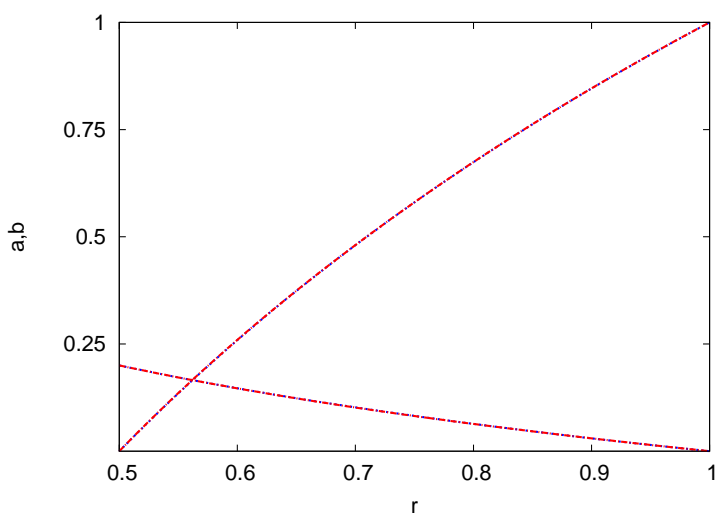

(a)

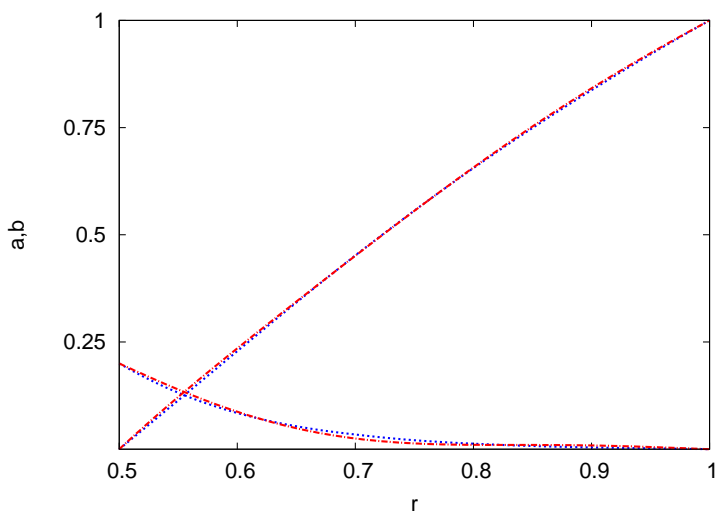

(b)

Figure 2. (Color online) Steady-state reactant and autocatalyst concentration profiles, $a$ and $b$ versus $r$. The parameters are $r_{1}=0.5, b_{0}=0.2$ and $\gamma=0.05$, with $\beta=40$ in (a) and $\beta=2000$ in (b). The two-term (red large dashes) semi-analytical solutions and the numerical solution of (2) (blue small dashes) are shown.

numerical steady-state solutions exist beyond these points, on these branches. It can be seen that the two-term solution is very close to the numerical solution of the pdes with less than $6 \%$ error.

Figure 4 shows steady-state diagrams for two different annuli. The inner radius $r_{1}=0.1$ in figure $4(\mathrm{a})$ and $r_{1}=0.25$ in figure $4(\mathrm{~b})$. The other parameters are $b_{0}=0.25, \gamma=0.03$. The one-term and two-term semi-analytical solutions plus the numerical solution of (2) are shown. For $r_{1}=0.1$ the breaking wave pattern occurs while for $r_{1}=0.25$, a thinner annulus, a unique pattern occurs. Hence reducing the thickness of the annulus eliminates the multiple steadystate solutions. The numerical bifurcation points shown in figure $4(\mathrm{a})$ are $(\beta, b)=(108,0.107)$ and $(105,0.275)$ and the two-term semi-analytical bifurcation points are $(\beta, b)=(111,0.133)$ and $(108,0.241)$. The two-term approximation is very close to the numerical solution of the pdes. At $\beta=300$ the error of the two-term solution is about $1 \%$, while for the one term approximation, the error is about $12 \%$.

\section{Singularity theory}

Singularity theory is a theoretical technique to analyse the distinct steady-state behaviour of a set of odes in a comprehensive manner, see Gray and Roberts [20]. The application of singularity theory to chemical reactions is described in Balakotaiah and Luss [21]. Here we apply singularity theory to the semi-analytical ode model derived in $\S 2$. This analysis provides semi-analytical parameter maps for each of the generic types of bifurcation pattern that can occur. The equations corresponding to the steady-state, two-term model, have the general form

$$
f_{i}\left(b_{0}, b_{1}, b_{2}, a_{1}, a_{2}, \beta, \gamma\right)=0, \quad i=1 \ldots 4,
$$

where $\beta$ is the bifurcation parameter. For the hysteresis bifurcation pattern a hysteresis loop occurs in the steady-state diagram, which corresponds to

$$
\frac{d \beta}{d b_{1}}=\frac{d^{2} \beta}{d b_{1}^{2}}=0 .
$$

The equations (5) only give an implicit relationship for $\beta$ so the conditions (6) are applied by using implicit differentiation. The parameters $\gamma$ and $b_{0}$ are constant while the first condition of (6) states $\frac{d \beta}{d b_{1}}=0$. The total derivative of (5) with respect to $b_{1}$ is

$$
\frac{d f_{i}}{d b_{1}}\left(b_{1}, b_{2}, a_{1}, a_{2}\right)=f_{i_{b_{1}}}+f_{i_{b_{2}}} \frac{d b_{2}}{d b_{1}}+f_{i_{a_{1}}} \frac{d a_{1}}{d b_{1}}+f_{i_{a_{2}}} \frac{d a_{2}}{d b_{1}}=0, \quad i=1 \ldots 4 .
$$

In (7) the $f_{i}$ are written as functions of $b_{1}, b_{2}, a_{1}$ and $a_{2}$. This notation implies that the $f_{i}$ depend on $b_{1}$ both explicitly and implicitly via $b_{2}, a_{1}$ and $a_{2}$. The second total derivative of (5) and conditions (6) give

$$
\frac{d^{2} f_{i}}{d b_{1}^{2}}\left(b_{1}, b_{2}, a_{1}, a_{2}\right)=0, \quad i=1 \ldots 4 .
$$

The two-term hysteresis bifurcation points are given by (5), (7) and (8), which represent twelve equations in the fifteen variables $b_{0}, b_{1}, b_{2}, a_{1}, a_{2}, \beta, \gamma, b_{0}, \frac{d b_{2}}{d b_{1}}, \frac{d^{2} b_{2}}{d b_{1}^{2}}, \frac{d a_{1}}{d b_{1}}, \frac{d^{2} a_{1}}{d b_{1}^{2}}, \frac{d a_{2}}{d b_{1}}, \frac{d^{2} a_{2}}{d b_{1}^{2}}$ and $r_{1}$. Six new variables have been introduced into 


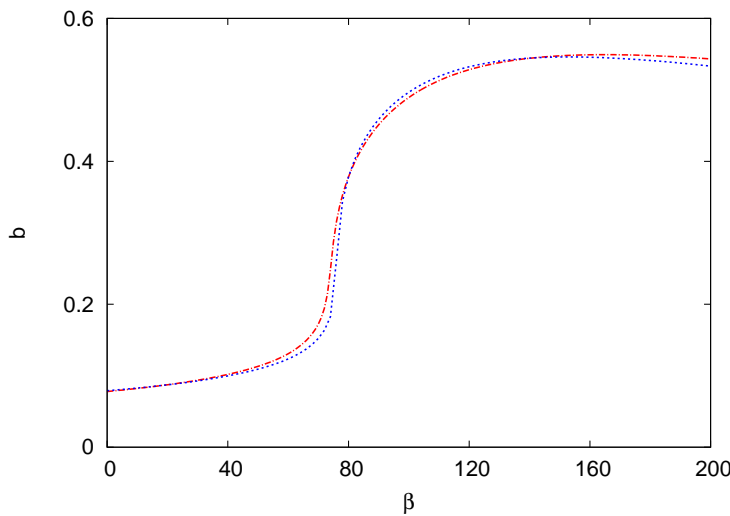

(a)

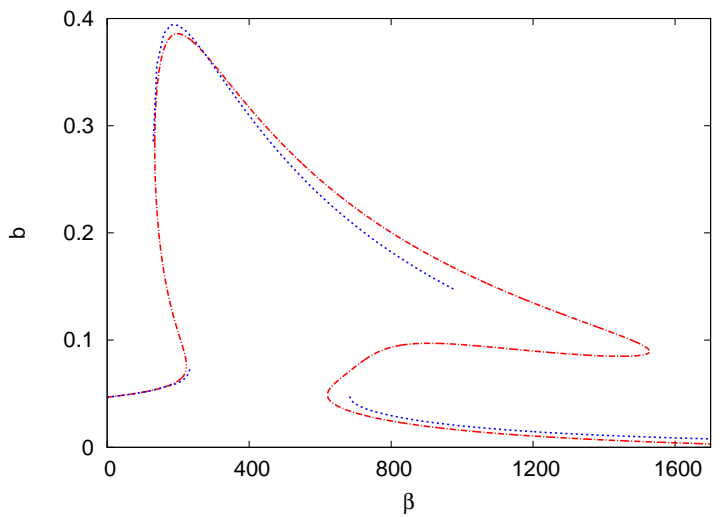

(c)

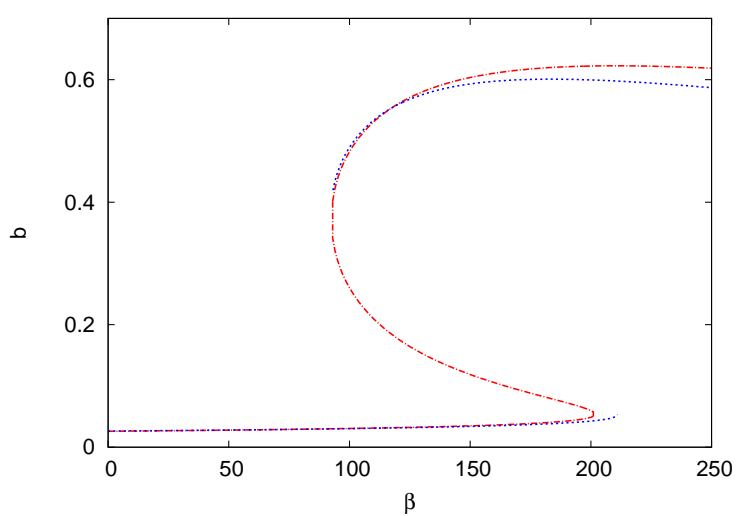

(b)

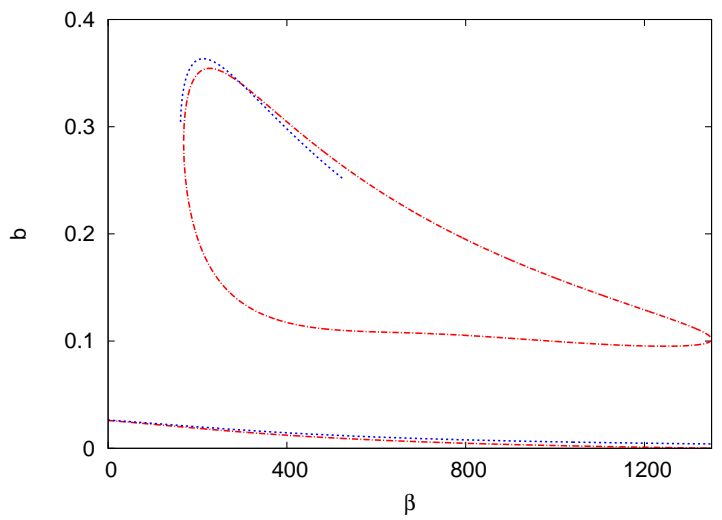

(d)

FiguRE 3. (Color online) Bifurcation diagrams; the autocatalyst concentration, $b$ versus $\beta$ for (a) $r_{1}=0.1$ the unique pattern with $b_{0}=0.3$ and $\gamma=0.02$; (b) the breaking-wave pattern with $b_{0}=0.1$ and $\gamma=0.01$; (c) the mushroom pattern with $b_{0}=0.12$ and $\gamma=0.018$; and (d) the isola pattern with $b_{0}=0.03$ and $\gamma=0.015$. The concentration $b$ is at $r=r_{c}$. The one-term (black solid lines) and two-term (red large dashes) semi-analytical solutions plus the numerical solution of (2) (blue small dashes) are shown.

the system, as a consequence of the implicit differentiation. We consider fixed values of $r_{1}$ and consider the loci of points in the $\gamma-b_{0}$ plane. The hysteresis points represent a line in the $\gamma-b_{0}$ plane which, when crossed, causes a hysteresis loop to be created or destroyed in the steady-state diagram. Maple is used solve the equations for the hysteresis bifurcation points. A similar method is used to translate the conditions for the isola bifurcation curve, the double-zero eigenvalue (DZE) and transversality Hopf degeneracies into sets of transcendental equations. The isola bifurcation points are defined by

$$
\frac{d \beta}{d b_{1}}=\frac{d \gamma}{d \beta}=0
$$

Crossing the degenerate isola curves leads to the creation or destruction of an isola, in the bifurcation pattern.

\subsection{The effect of varying the thickness of the annulus}

In this section we consider the effect of varying the radius of the inner edge of the annulus $r_{1} \in(0,1)$. The semi-analytical model can be used to quantify the transitions in the number of steady-state diagrams, and changes to parameter regions, as the value of $r_{1}$ is varied. This however presents significant computational challenges as the ode model changes (see Equations (12)-(15) for the case of $\left.r_{1}=0.25\right)$ as $r_{1}$ is varied. In order to analyze the effects of varying $r_{1}$ twenty five different ode models were developed for $r_{1}=[0.01,0.99]$ at steps of $r_{1} \approx 0.04$. All of these ode models were solved to 


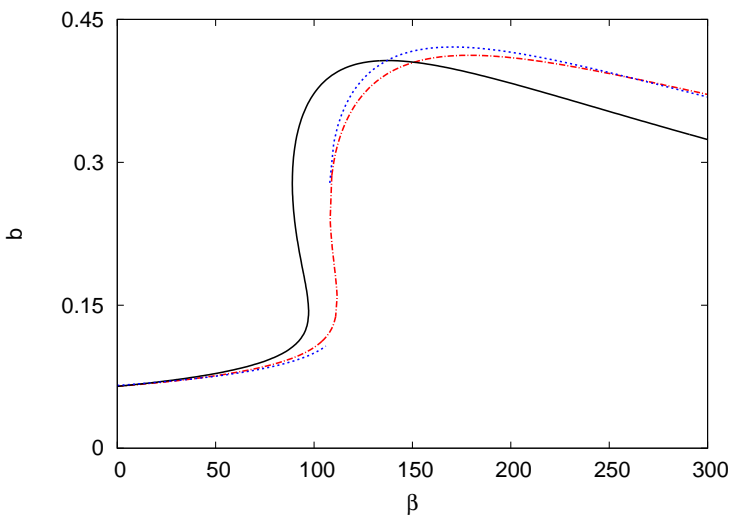

(a)

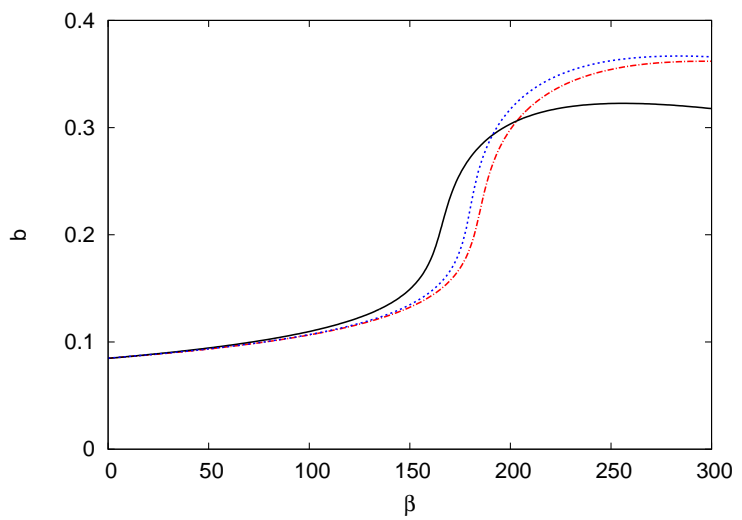

(b)

Figure 4. (Color online) Bifurcation diagrams; the autocatalyst concentration, $b$ versus $\beta$ for (a) $r_{1}=0.1$ the breaking-wave pattern, (b) $r_{1}=0.25$ the unique pattern. The other parameters are $b_{0}=0.25, \gamma=0.03$. The concentration $b$ is at $r=r_{c}$. The one-term (black solid lines) and two-term (red large dashes) semi-analytical solutions plus the numerical solution of (2) (blue small dashes) are shown.

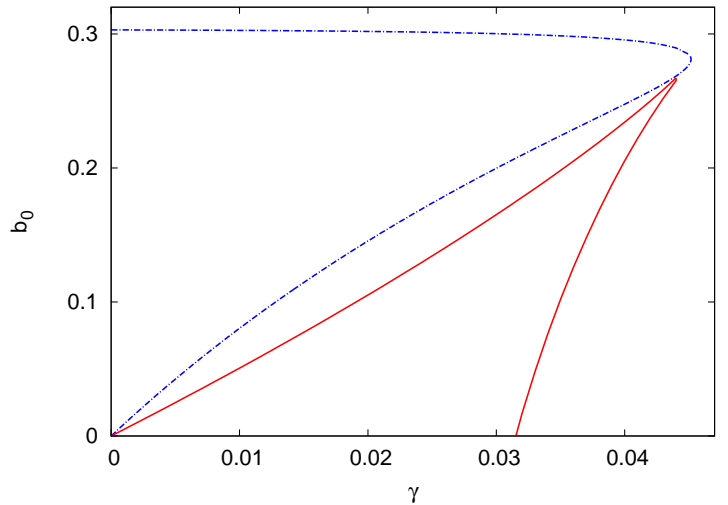

(a)

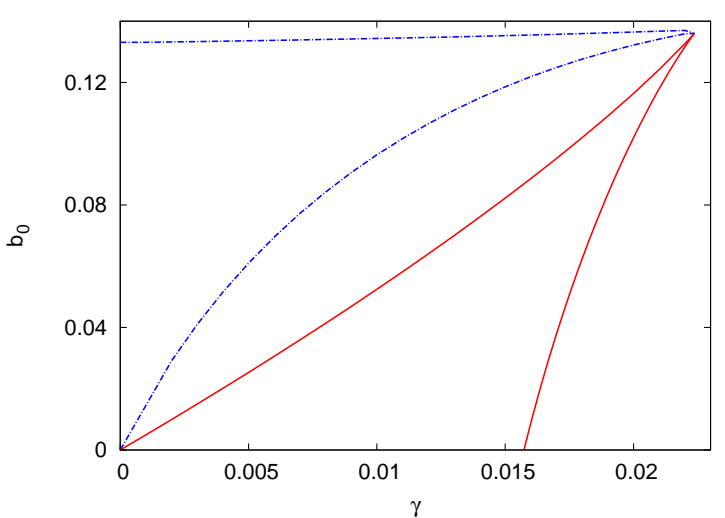

(b)

Figure 5. (Color online) Division of the $\gamma$ - $b_{0}$ plane into regions corresponding to the different bifurcation diagrams, (a) $r_{1}=0.1$ and (b) $r_{1}=0.9$. The isola curves (red solid lines) and the hysteresis curves (blue small dashes) are shown

find their hysteresis and isola curves and the various intersection and maximum points. These points were then plotted and a curve fitting routine used to obtain the smooth curves shown in figures 6 and 7 below.

Figure 5 shows the division of the $\gamma$ - $b_{0}$ plane into regions corresponding to the different steady-state diagrams for (a) a thick annulus $r_{1}=0.1$, and (b) a thin annulus $r_{1}=0.9$. As the thickness of the reactor, $1-r_{1}$, varies then significant changes in the bifurcation regions also occur. In this geometry, as the annulus becomes thinner, changing from a thickness of $1-r_{1}=0.9$ to $1-r_{1}=0.1$, the regions of parameter space in which multiple steady-state solutions occur become much smaller. Also note that in the limit $r_{1} \rightarrow 1$, the dynamics of the annulus reactor would be similar to that of an equivalent 1-D reaction-diffusion slab cell, as the curvature effect are small. Figure 6(a) shows the intersection point between the isola curve and the $\gamma$-axis, versus $r_{1}$. This is a measure of the width of the parameter region in which multiple steady-state solutions occur. As the value of $r_{1}$ increases, the value of $\gamma$ decreases, so that the region of parameter space in which multiple steady-state solutions occur is reduced. Figure 6(b) shows the intersection point between the cusp curve and the $b_{0}$-axis versus $r_{1}$, which is a measure of the height of the parameter space. As the value of $r_{1}$ increases, the value of $b_{0}$ decreases, again reducing the parameter space. Figure 7 shows the maximum point of 


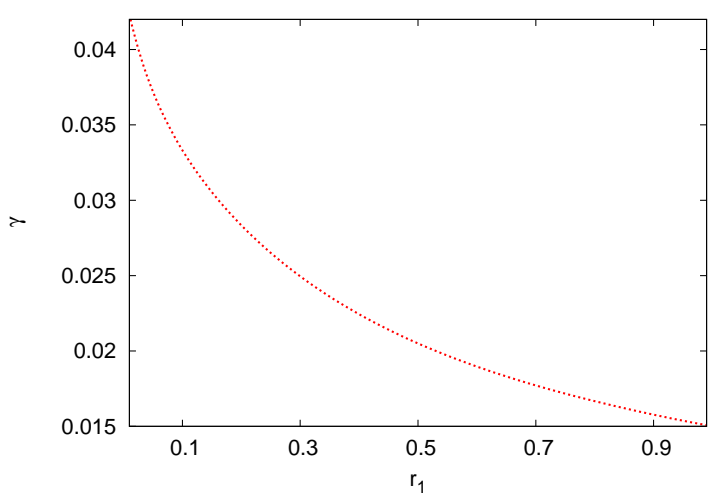

(a)

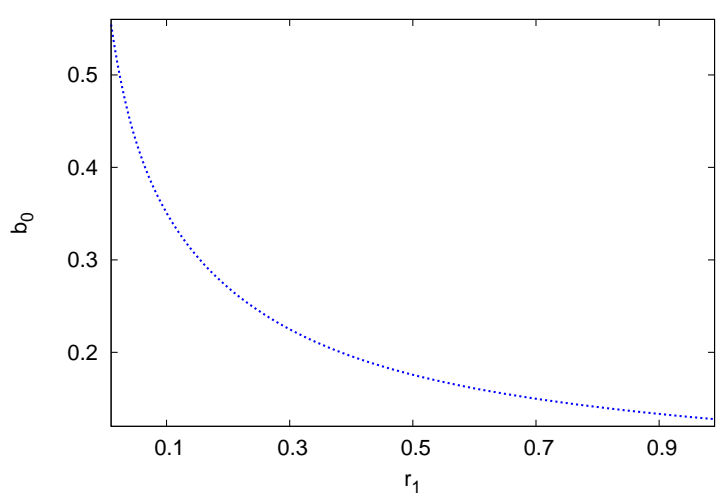

(b)

Figure 6. (Color online) The intersection points between (a) the isola curves and the $\gamma$-axis and (b) the cusp curves and $b_{0}$-axis, versus $r_{1}$.

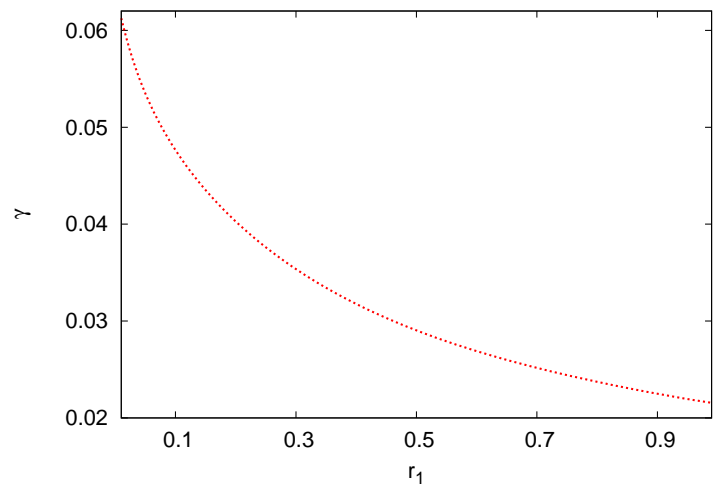

(a)

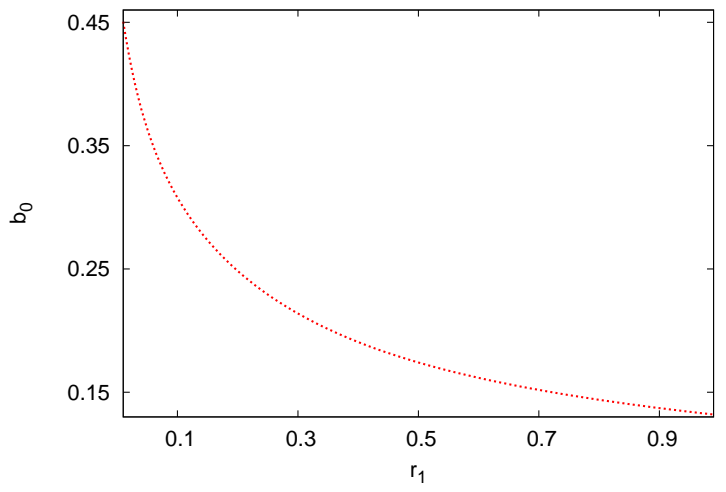

(b)

Figure 7. (Color online) The maximum point of the isola curve, (a) $\gamma$ versus $r_{1}$, and (b) $b_{0}$ versus $r_{1}$.

the isola curve, (a) $\gamma$ versus $r_{1}$ and (b) $b_{0}$ versus $r_{1}$. As the value of $r_{1}$ increases, the values of $b_{0}$ and $\gamma$ decrease, and the regions of parameter space is reduced. Thus a general trend is that a thin annulus has a smaller region of parameter space in which the various types of bifurcation diagrams occur, which represent diagrams with multiple steady-state solutions.

\section{Local stability and oscillatory solutions}

Hopf bifurcations and oscillatory solutions are classical phenomena which occur in many chemical and biological systems. The theory of Hopf bifurcations is explained in many texts on bifurcation theory and dynamical systems, see for example Guckenheimer and Holmes [22] or Golubitsky and Schaeffer [23]. Hopf bifurcations also occur in a 1-D Gray-Scott reaction-diffusion cell; Gray and Scott [4] considered the local stability by calculating the Jacobian matrix of the finitedifference form of the pdes while Marchant [8] considered the stability of a lower order ode model. In both approaches a region of the $\gamma-b_{0}$ plane, in which Hopf bifurcations can occur, was identified. Here the stability of the semi-analytical model (2) is considered. The Hopf degeneracy points are calculated to find a semi-analytical parameter map in which Hopf bifurcations occur, for the reaction-diffusion annulus. For the one-term model, $a_{2}=b_{2}=0$, the semi-analytical 


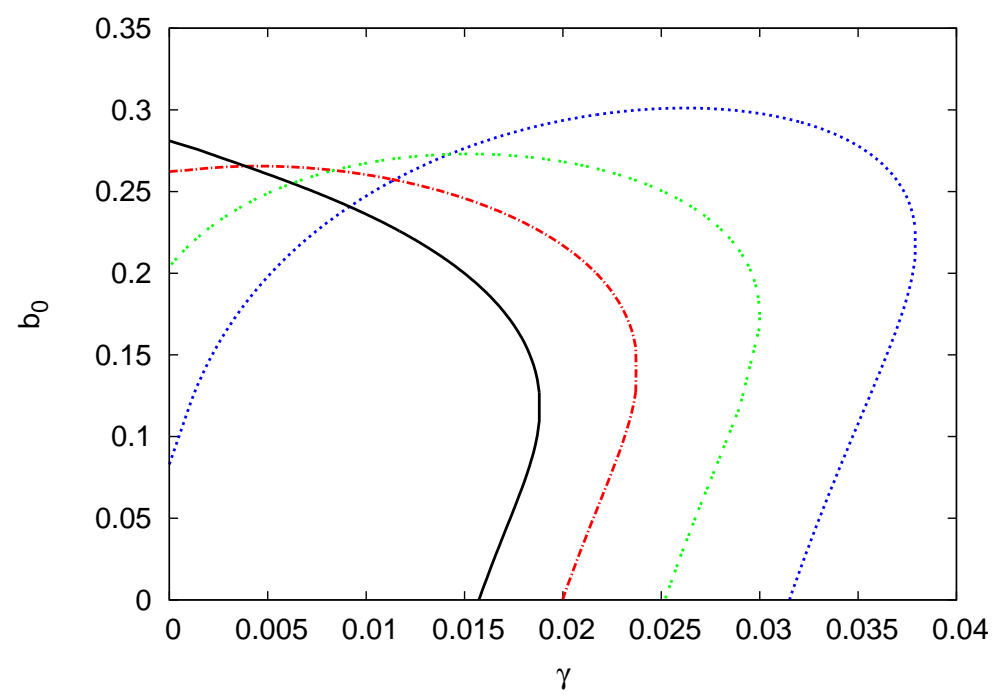

Figure 8. (Color online) The double Hopf curve when $r_{1}=0.1$ (small blue dashes), $r_{1}=0.25$ (green double dashes), $r_{1}=0.5$ (large red dashes) and when $r_{1}=0.9$ (black solid line).

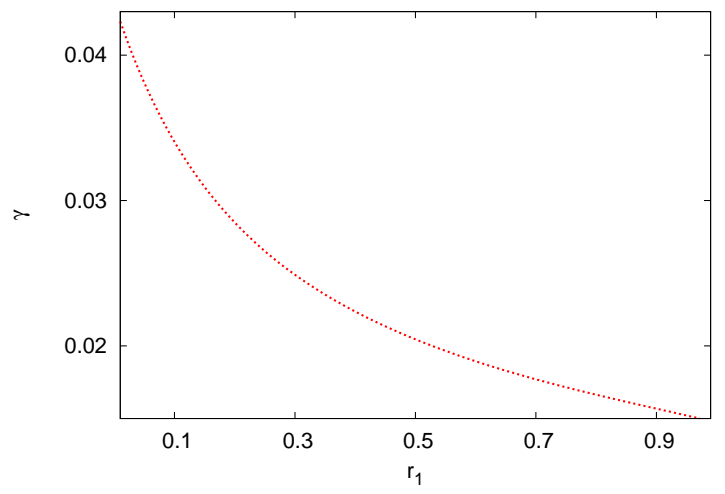

(a)

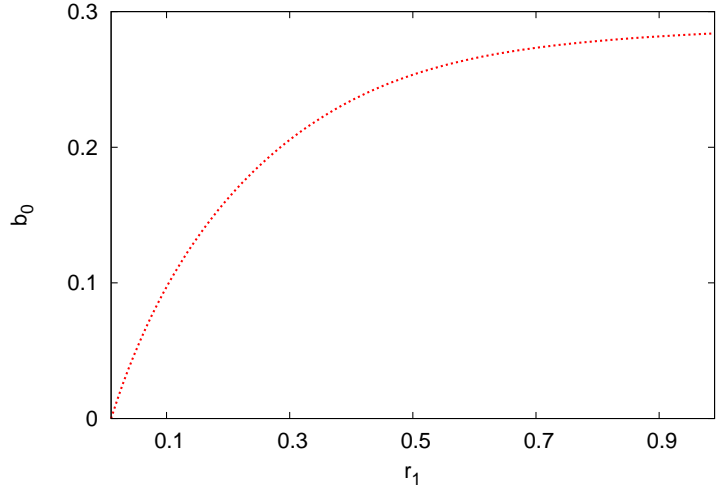

(b)

Figure 9. (Color online) The intersection points between (a) the Hopf curve and the $\gamma$-axis and (b) the Hopf curve and $b_{0}$-axis, versus $r_{1}$.

model is given by two odes for $a_{1}$ and $b_{1}$. The degenerate Hopf points are defined by

$$
D Z E: f_{i}=\operatorname{tr} \mathrm{J}=\operatorname{det} \mathrm{J}=0, \quad \mathrm{H} 2: \mathrm{f}=\operatorname{tr} \mathrm{J}=\frac{\mathrm{d} \operatorname{tr} \mathrm{J}}{\mathrm{d} \beta}=0, \mathrm{i}=1,2 .
$$

For the two-term semi-analytical model, the set of equations expands to four odes. The eigenvalues of the Jacobian matrix are described by the quartic equation $\lambda^{4}+\alpha_{1} \lambda^{3}+\alpha_{2} \lambda^{2}+\alpha_{3} \lambda+\alpha_{4}=0$. Hopf bifurcations occur for this system when one pair of eigenvalues is purely imaginary, which implies $q=\alpha_{4} \alpha_{1}^{2}+\alpha_{3}^{2}-\alpha_{1} \alpha_{2} \alpha_{3}=0$. The degenerate Hopf points are given by

$$
D Z E: f_{i}=\alpha_{3}=\alpha_{4}=0, \quad H 2: f_{i}=q=\frac{d q}{d \beta}=0, i=1,4 .
$$

By solving the DZE and H2 conditions, we obtain curves in the $\gamma-b_{0}$ plane. We will discuss the effect of varying the thickness of the annulus on the occurrence of Hopf bifurcations. 
Figure 8 shows the double Hopf curve in the $\gamma-b_{0}$ plane for $r_{1}=0.1,0.25,0.5$ and 0.9 . Only the H2 Hopf curve is shown as the DZE curve always lies inside the H2 curve. The maximum heights are $b_{0} \approx 0.301,0.273,0.265$ and 0.281 for $r_{1}=0.1,0.25,0.5$ and 0.9 respectively. Hence the height of the bifurcation pattern varies in a non-monotonic manner. The intersection points of the Hopf curve and $b_{0}$-axis are $b_{0}=0.083,0.205,0.262$ and 0.281 , while the intersection points of the Hopf curve and $\gamma$-axis are $\gamma=0.031,0.025,0.02$ and 0.016 . Hence, Hopf points are found in a larger region of parameter space for a thick annulus, which corresponds to small values of $r_{1}$. However for a thin annulus, for small values of $\gamma$, there is an increased parameter space in which Hopf bifurcation points occur.

Figure 9 shows the intersection points between the Hopf curves and (a) the $\gamma$-axis, (b) the $b_{0}$-axis, versus $r_{1}$. The height of the Hopf curve increases near its intersection with the $b_{0}$-axis as $r_{1}$ increases, and its extent shrinks near the $\gamma$-axis as $r_{1}$ increases. Hence large value of $r_{1}$, which corresponds to a thin annulus, leads to an increased possibility of Hopf bifurcations and oscillatory solutions near the $b_{0}$-axis as shown in figure $9(\mathrm{~b})$, and a decreased possibility near the $\gamma$-axis, as shown in figure 9 (a). The technique which is used to obtain values of figure 9 is similar to that for figure 6 and 7 . Many ode models for differing $r_{1}$ were solved to find the $\mathrm{H} 2$ curve and the intersection points. These points were all plotted to obtain the smooth curves of figure 9.

Figure 10(a) shows a limit cycle curve, $a$ versus $b$, while 10(b) and 10(c) show the time evolution of the reactant and autocatalyst, $a$ and $b$ versus t respectively. The parameters are $b_{0}=0.25, \gamma=0.031, \beta=1000$ and $r_{1}=0.1$. The parameter choice used here lie to the left of the $r_{1}=0.1$ Hopf curve in figure 8 . Hence the occurrence of limit cycles in this example is consistent with the theoretical prediction of the ode model. The numerical solutions of (2) are shown where the period of the limit cycle is 0.884 while, the amplitudes of the limit cycle are 0.606 and 0.234 for the reactant and autocatalyst concentrations respectively. Figure 11 shows (a) the reactant and (b) the autocatalyst concentrations, $a$ and $b$ versus $t$. The parameters are the same as for figure 10 except that $r_{1}=0.25$. The parameters lie to the right of the $r_{1}=0.25$ Hopf curve in figure 8. Hence the solution here is stabilized by the thinner annulus geometry, as predicted by the ode based theory. The one term, two-term semi-analytical solutions and the numerical solutions of (2) are shown. After some initial relaxation oscillations the solutions evolve to the steady-states for large time, with $a \rightarrow 0.039$ and $b \rightarrow 0.192$ as $t \rightarrow \infty$. The two-term expression gives a good approximation when compared with the numerical solution of the governing pdes. At $t=3$, for two-term semi-analytical approximations, the errors are less than $2 \%$.

\section{Conclusion}

Semi-analytical solutions have been developed for the Gray-Scott scheme in a reaction-diffusion annulus. The effect of varying the thickness of the annulus has been considered and the model is shown to exhibit a wide variety of complexity, both for bifurcation patterns and Hopf bifurcation points. In general, a thicker annulus will display multiple steady-state and Hopf bifurcations in a larger region of parameter space, than a thin annulus. Comparisons with numerical solutions show that the two-term semi-analytical model is very accurate. A wide range of future work is possible. Symmetry breaking Hopf bifurcations and the existence and stability of a rotating wave in an annulus reactor could be considered. Also chemical schemes with experimental applicability could be analysed in a reaction-diffusion annulus, such as the BZ or glycolytic reaction schemes.

\section{Appendix: The ode model for inner radius $r_{1}=0.25$}

$$
\begin{aligned}
\frac{d}{d t} a_{1}=\quad & -0.504 \beta a_{2} b_{0} b_{2}-0.0766 \beta b_{0} b_{2}-0.405 \beta b_{0} b_{1}-0.213 \beta a_{2} b_{0} b_{1}-0.427 \beta b_{2}^{2}-0.5 \beta a_{1} b_{2}^{2} \\
& -0.55 \beta b_{1}^{2}-0.75 \beta a_{1} b_{1}^{2}-0.599 \beta a_{1} b_{0} b_{1}-16.0 a_{1}-5.20 a_{2}-0.098 b_{0}^{2} \\
& -0.155 \beta a_{1} b_{0}^{2}-0.213 \beta a_{1} b_{0} b_{2}-\beta a_{2} b_{1} b_{2}+0.213 \beta b_{1} b_{2}-0.123 \beta a_{2} b_{0}^{2} \\
\frac{d}{d t} a_{2}=\quad & -0.383 \beta a_{2} b_{0} b_{2}-0.351 \beta b_{0} b_{2}-0.504 \beta a_{2} b_{0} b_{1}-0.5 \beta a_{2} b_{1}^{2}+0.192 \beta b_{2}^{2} \\
& -0.75 \beta a_{2} b_{2}^{2}-0.077 \beta b_{0} b_{1}+0.107 \beta b_{1}^{2}-0.213 \beta a_{1} b_{0} b_{1}+6.63 a_{1}-68.4 a_{2} \\
& -0.067 \beta b_{0}^{2}-0.124 \beta a_{1} b_{0}^{2}-0.855 \beta b_{1} b_{2}-\beta a_{1} b_{1} b_{2}-0.504 \beta a_{1} b_{0} b_{2}-0.203 \beta a_{2} b_{0}^{2} \\
\frac{d}{d t} b_{1}=\quad & 0.504 \beta a_{2} b_{0} b_{2}+0.077 \beta b_{0} b_{2}+0.213 \beta a_{2} b_{0} b_{1}+0.405 \beta b_{0} b_{1}+0.427 \beta b_{2}^{2}+0.5 \beta a_{1} b_{2}^{2} \\
& +0.55 \beta b_{1}^{2}+0.75 \beta a_{1} b_{1}^{2}+0.213 \beta a_{1} b_{0} b_{2}+0.599 \beta a_{1} b_{0} b_{1}+0.098 \beta b_{0}^{2}+0.155 \beta a_{1} b_{0}^{2} \\
+ & \beta a_{2} b_{1} b_{2}+0.124 \beta a_{2} b_{0}^{2}-16.0 b_{1}-0.466 \gamma \beta b_{0}-\gamma \beta b_{1}-5.20 b_{2}-0.213 \beta b_{1} b_{2} \\
& 0.383 \beta a_{2} b_{0} b_{2}+0.351 \beta b_{0} b_{2}+0.504 \beta a_{2} b_{0} b_{1}+0.0766 \beta b_{0} b_{1}+0.5 \beta a_{2} b_{1}^{2}-0.192 \beta b_{2}^{2} \\
\frac{d}{d t} b_{2}=\quad & 0.75 \beta a_{2} b_{2}^{2}-0.107 \beta b_{1}^{2}+0.213 \beta a_{1} b_{0} b_{1}+0.067 \beta b_{0}^{2}+0.124 \beta a_{1} b_{0}^{2}+0.855 \beta b_{1} b_{2} \\
+ & +\beta a_{1} b_{1} b_{2}-68.4 b_{2}+0.504 \beta a_{1} b_{0} b_{2}+0.203 \beta a_{2} b_{0}^{2}+76.6 b_{1}-0.290 \gamma \beta b_{0}-\gamma \beta b_{2}
\end{aligned}
$$




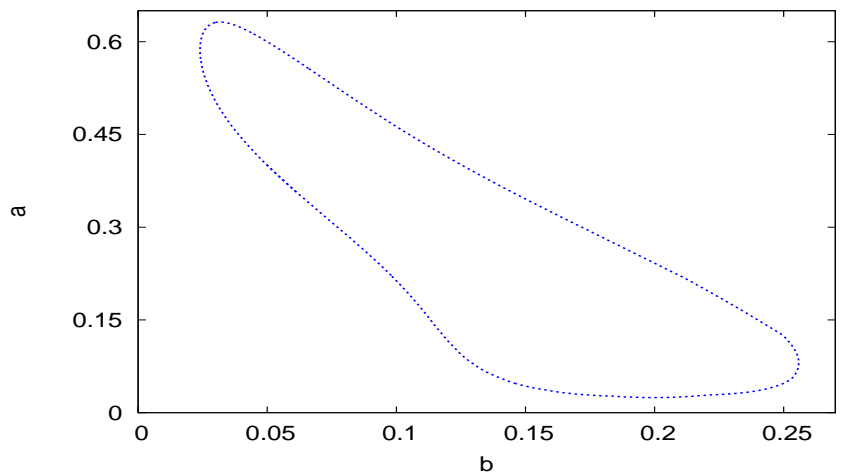

(a)

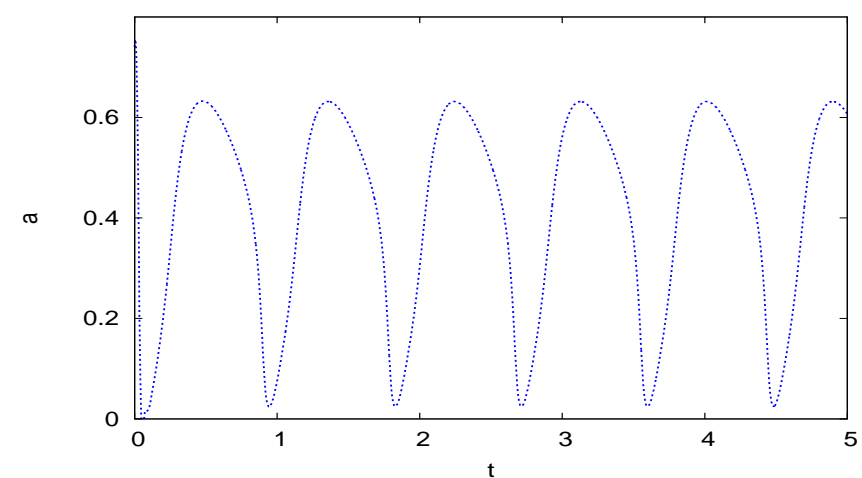

(b)

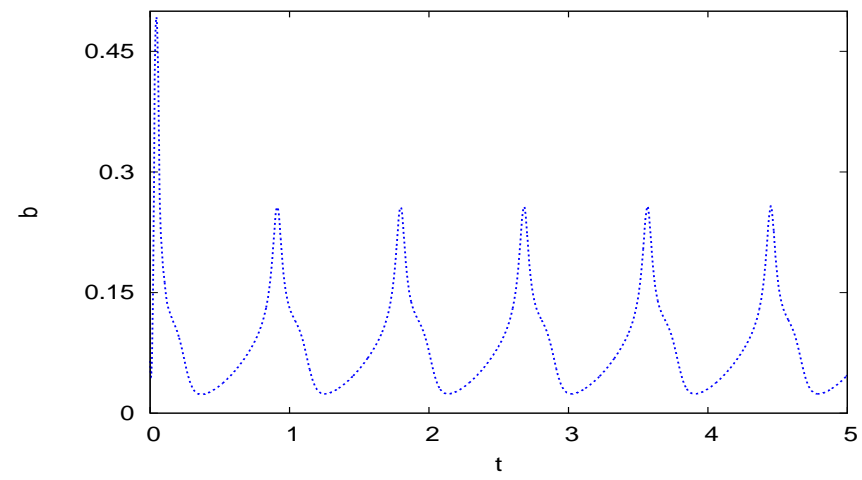

(c)

Figure 10. (Color online) Limit cycle curve, reactant and autocatalyst concentrations, $a$ versus $b$. The parameters are $b_{0}=0.25, \gamma=0.031, \beta=1000$, and $r_{1}=0.1$.

\section{References}

[1] J. M. L. Corbel, J. N. J. Van Lingen, J. F. Zevenbergen, O. L. J. Gijzeman, A. Meijerink, Strobes: pyrotechnic compositions that show a curious oscillatory combustion, Angew. Chem. Int. Ed. 52 (2013) 290-303.

[2] F. Sagues, I. R. Epstein, Nonlinear chemical dynamics, Dalton. Trans. 7 (2003) 1201-1217.

[3] P. Gray, S. K. Scott, Autocatalytic reactions in the isothermal continuous, stirred tank reactor: isolas and other forms of multistability, Chem. Engng. Sci. 38 (1983) 29-43.

[4] P. Gray, S. K. Scott, Autocatalytic reactions in the isothermal continuous, stirred tank reactor: oscillations and instabilities in the system $\mathrm{a}+2 \mathrm{~b}$ to $3 \mathrm{~b}$; b to c, Chem. Engng. Sci. 39, (1984) 1087-1097. 


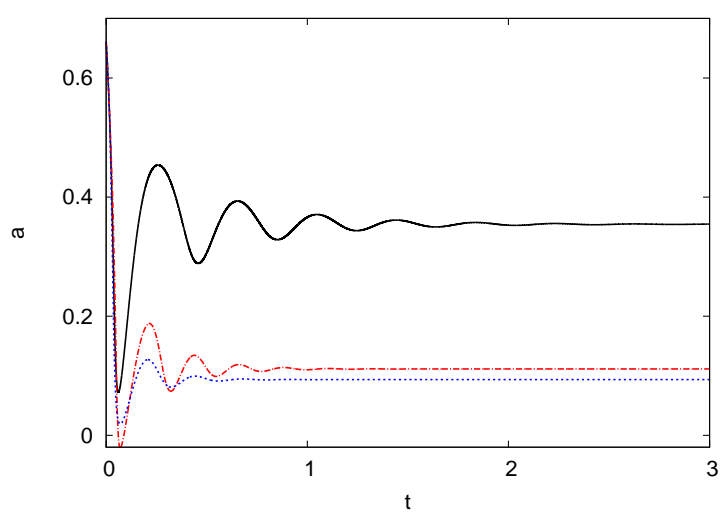

(a)

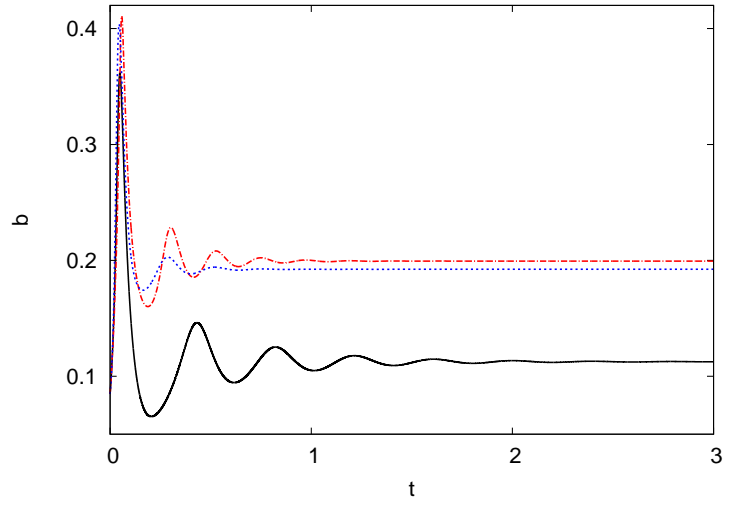

(b)

Figure 11. (Color online) The reactant and autocatalyst concentrations, $a$ and $b$ versus $\mathrm{t}$ in (a) and (b) respectively. The parameters are $b_{0}=0.25, \gamma=0.031, \beta=1000$ and $r_{1}=0.25$. The one-term (black solid line), the two-term (red large dashes) semianalytical solutions and the numerical solution of (2) (blue small dashes) are shown.

[5] S. R. Kay, S. K. Scott, P. G. Lignola, The application of singularity theory to isothermal autocatalytic reactions: the influence of uncatalysed reactions, Proc. Roy. Soc. Lond A 409, (1987) 433-448.

[6] S. K. Scott, Isolas, mushrooms and oscillations in isothermal, autocatalytic reaction-diffusion equations, Chem. Engng. Sci. 42 (1987) 307-315.

[7] S. R. Kay, S. K. Scott, Multiple stationary states, sustained oscillations and transient behavior in autocatalytic reaction-diffusion equations, Proc. Roy. Soc. Lond A 418 (1988) 345-364.

[8] T. R. Marchant, Cubic autocatalytic reaction-diffusion equations:semi-analytical solutions, Proc. Roy. Soc. Lond A 458 (2002) 873-888.

[9] W. Tam, W. Horstemke, Z. Noszticzius, H. L. Swinney, Sustained sprial waves in a continuously fed unstrirred chemical reactor, J. Chem. Phys. 88 (1988) 3395-3396.

[10] S. Bagyan, T. Mair, E. Dulos, J. Boissonade, P. DeKepper, S. Muller, Glycolytic oscillations and waves in an open spatial reactor: Impact of feedback regulation of phosphofructokinase, Biophys. Chem. 116 (2005) 67-76.

[11] A. Lavrova, S. Bagyan, T. Mair, M. Hauser, L. Schimansky-Geier, Modeling of glycolytic wave propagation in an open spatial reactor with inhomogeneous substrate flux, Biosystems 97 (2009) 127-133.

[12] M. J. Chaplain, M. Ganesh, I. G. Graham, Spatio-temporal pattern formation on spherical surfaces: numerical simulation and application to solid tumour growth, J. Math. Biol. 42 (2001) 387-423.

[13] T. Roose, S. J. Chapman, P. K. Maini, Mathematical models of avascular tumor growth, SIAM Rev. 49 (2007) 179-208.

[14] Q. Nie, Y. T. Zhang, R. Zhao, Efficient semi-implicit schemes for stiff systems, J. Comput. Phys. 214 (2006) $521-537$.

[15] L. Jiangguo, T. Simon, Semi-implicit spectral collocation methods for reaction-diffusion equations on annuli, Numer. Meth. Part. Diff. Eq. 27 (2011) 1113-1129.

[16] W. W. Farr, M. Golubitsky, Rotating chemical waves in the Gray-Scott model, SIAM J. Appl. Math. 52 (1992) $181-221$.

[17] S. Lubkin, R. Rand, Oscillatory reaction-diffusion equations on rings, J. Math. Biol. 32 (1994) $617-632$.

[18] M. Bar, A. K. Bangia, I. G. Kevrekidis, Bifurcation and stability analysis of rotating chemical spirals in circular domains: boundary-induced meandering and stabilization, Phys. Rev. E 67 (2003) 056126.

[19] J. C. Tsai, Rotating spiral waves in lambda-omega systems on circular domains, Physica D 239 (2010) $1007-1025$.

[20] B. G. Gray, M. J. Roberts, A method for the complete qualitative analysis of two coupledordinary differential equations dependent on three parameters, Proc. Roy. Soc. Lond A 416 (1988) 361-389.

[21] V. Balakotaiah, D. Luss, Multiplicity features of reacting systems, Chem. Engng. Sci. 38 (1983) 1709-1721.

[22] J. Guckenheimer, P. Holmes, Nonlinear oscillations, dynamical systems, and bifurcations of vector fields, SpringerVerlag, New York, 1983.

[23] M. Golubitsky, D. G. Schaeffer, Singularites and groups in bifurcation theory, Springer-Verlag, New York, 1985. 